\title{
Frequency Analysis of Power Consumption in Agitation Granulation of Powder Materials with Sparingly Soluble Acetaminophen
}

\author{
Satoru Watano,* Keijiro Terashita and Kei Miyanami \\ Department of Chemical Engineering, University of Osaka Prefecture, Mozu-Umemachi, Sakai, Osaka 591, Japan. Received June 19, 1991
}

\begin{abstract}
Frequency analysis of power consumption obtained during the agitation granulation process was performed by using powder materials with sparingly soluble acetaminophen. The granulation process and granule growth were evaluated by means of granule properties such as mean particle diameter and geometric standard deviation. In accordance with these properties to the temporal behavior of the intensity of spectrum calculated by the FFT (fast Fourier transform) analysis and the coefficient of variation (C.V. value) computed from the power consumption data, the granulation mechanism and optimal operational end-point were determined. On the basis of the fact that this practical method is applicable to the powder materials composed of the sparingly soluble acetaminophen which is difficult to make granule by wet granulation, adaptability and universality were proven.
\end{abstract}

Keywords acetaminophen; frequency analysis; FFT (fast Fourier transform); power consumption; agitation granulation; operation end point

\section{Introduction}

In the preceding paper, ${ }^{1)}$ we suggested that the frequency analysis of power consumption was applicable to the determination of the operational end point in the agitation granulation process. The report dealt with powder materials without any ingredients. In order to guarantee the universality of this method, acetaminophen, which has been thought to be difficult to make into granules by molding because of its sparingly soluble characteristics, was chosen as a component in powder materials. Power consumption was continuously measured during the granulation process, from which the calculation of $\mathrm{C}$. V. value (coefficient of variation) was conducted and the spectrum distributed frequency region was computed by using FFT (fast Fourier transform) analysis. The relation between the granule properties (the mean particle diameter and geometric standard deviation) and the temporal change of the C.V. value and the spectrum was discussed. On the basis of these findings, it was found that this practical method was useful in the determination of the mechanism and operational end point of the granulation process in using powder materials composed of a sparingly soluble ingredient such as acetaminophen, thus the universality of this analysis was confirmed.

\section{Experimental}

Materials Materials and mixing ratio in weight used are listed in Table I. (Here, mean particle diameter was measured by means of a rowtap method.) As the principal ingredient, acetaminophen, which has been difficult to produce in solid dosage form because of its sparingly soluble characteristics, was empolyed. The hydroxypropylcellulose-water

TABle I. Powder Component

\begin{tabular}{|c|c|c|}
\hline Components & $\begin{array}{l}\text { Mean particle diameter }{ }^{a)} \\
\qquad(\mu \mathrm{m})\end{array}$ & $\begin{array}{l}\text { Mixing ratio in weight } \\
(\mathrm{g})\end{array}$ \\
\hline Lactose $^{b)}$ & 104 & 100.8 \\
\hline Corn $\operatorname{starch}^{c)}$ & 42 & 43.2 \\
\hline Avicel $^{d)}$ & 76 & 12.0 \\
\hline Acetaminophenum ${ }^{e)}$ & 74 & 144.0 \\
\hline Total & & 300.0 \\
\hline
\end{tabular}

a) Determined by rowtap method. b) Pharmatose 200M, DMV. c) Corn Starch W, Nippon Shokuhin Kakou Co., Ltd. d) PH-101, Asahi Chemical Industry Co., Ltd. e) Daicel Chemical industries, Ltd. of $6.25 \%$ solution was used as a binder liquid. The total amount of $67.2 \mathrm{~g}$ of the binding solution was added at once to powder materials.

Apparatus and Method The granulation experiment was performed by a high speed type of granulator (LSF-GS-1J, Fukae Powtec) with an agitator rotating at $13.3 \mathrm{rps}$ and a chopper rotating at $8.33 \mathrm{rps}$. Power consumption of the agitator and chopper blade was measured by a power meter during the granulation process. The output electric signal from the power meter was digitalized by an $\mathrm{A} / \mathrm{D}$ converter and monitored and processed by a personal computer.

If there is no mention, the experimental condition and method in this study are the same as previously reported. ${ }^{1)}$

\section{Results and Discussion}

The required power consumption curve in the case of the acetaminophen is illustrated in Fig. 1. In Fig. 1, the middle curve indicates the power consumption of the agitator blade and the scattered plots near the time axis are of the chopper. The plots before $t=0$ is the value needed while mixing the dry material powder. Here, the power required to run the granulator idle was deducted from each measured value during the granulation process. In this study, in order to eliminate the interference of the electrical supply of the motor drive, an actual efficiency was adopted to the power consumption measurement system. The actual efficiency was calculated on the basis of the following equation.

actual efficiency $=$ circuit voltage $\times$ electric current $\times \cos \phi$

(Here, $\phi$ is a phase difference caused by the capacitive or inductive element in a circuit. The $\cos \phi$ is a power factor which indicates the ratio of effective power consumption to total power consumption).

On the basis of the measured power consumption data, if we adopt the method proposed by $\mathrm{K}$. Terashita and et

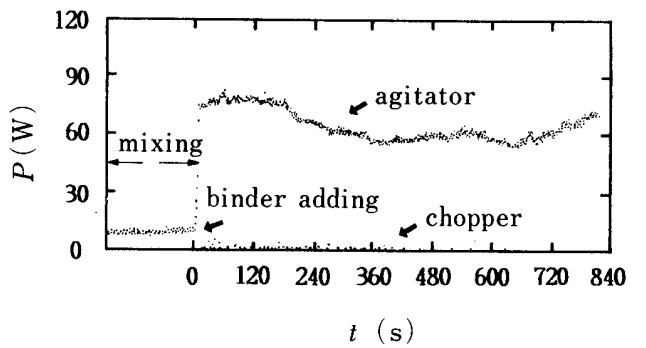

Fig. 1. Power Consumption, $P$ as a Function of Operation Time, $t$ 




Fig. 2. C.V. Value of Power Consumption as a Function of Granulation Time, $t$

$a .^{2)}$ to this powder materials, the operational end point can not be clearly evaluated because the power consumption obtained showed no steady state from the initial to the final stage of the granulation process. Thus, a frequency analysis $^{1)}$ was conducted in this system. The method of frequency analysis by FFT is the same as previously reported. ${ }^{1}$ In this paper, however, C.V. value of power consumption was newly introduced in order to get a dimentionless form of fluctuation.

Equations used for the calculation of the C.V. value were as follows; The mean difference of power consumption, $P$ is calculated as

$$
\bar{P}=\sum_{i=t}^{t+T} P_{i} / T
$$

Here, $P_{i}$ is the power consumption data at time $i$, and $T$ is the calculation interval (selected $30 \mathrm{~s}$ ). The mean deviation of power consumption fluctuations $\sigma$ is calculated by the following equation.

$$
\sigma=\left\{\sum_{i=t}^{t+T}\left(P_{i}-\bar{P}\right)^{2} /(T-1)\right\}^{0.5}
$$

The C.V. value is defined as

$$
\text { C.V. value }=\sigma / \bar{P}
$$

Figure 2 provides the C.V. value of the power consumption of the agitator blade as a function of granulation time. The power consumption of the chopper blade was so small that the calculation of the C.V. value was meaningless, thus it was abbreviated. The C.V. value of the agitator power consumption was large in the initial phase but decreased with the passage of granulation time. At the phase between $t=420$ and $540 \mathrm{~s}$, the minimum value was achieved. After that it showed an increase.

Figure 3 shows the spectrum obtained by the FFT analysis as a function of frequency and granulation time. The behavior of the spectrum appeared near at $5.4 \mathrm{~Hz}$ had the same tendency as that of the C.V. value, still more, it was found that by means of this analysis based on the FFT, the operational end point could be quantitatively determined, more accurately than that of the C.V. value. The frequency number where the peak merged was over a wide range at the initial phase, but converged to $5.4 \mathrm{~Hz}$. This specification frequency number was the same as the previous report, ${ }^{1}$ thus it was thought to be the number of proper vibration of this equipment and was unrelated to the kind of powder materials and experimental conditions.

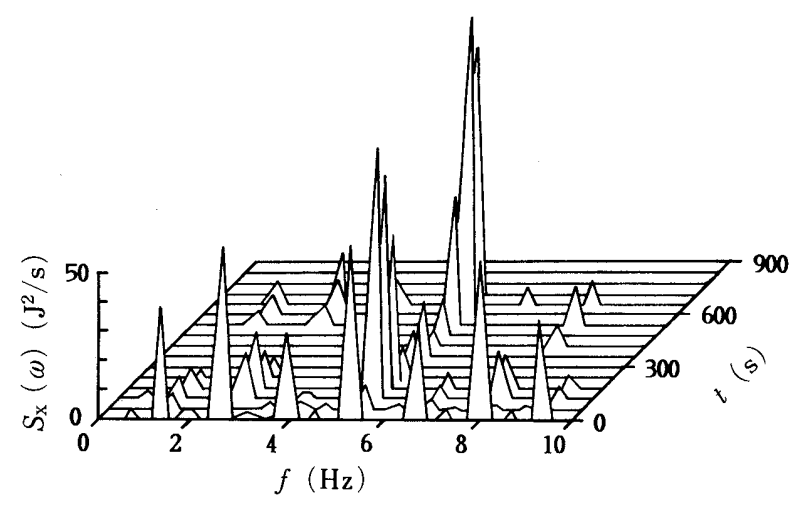

Fig. 3. Intensity of Spectrum, $S_{\mathbf{x}}(\omega)$ as a Function of Frequency, $f$ and Granulation Time, $t$

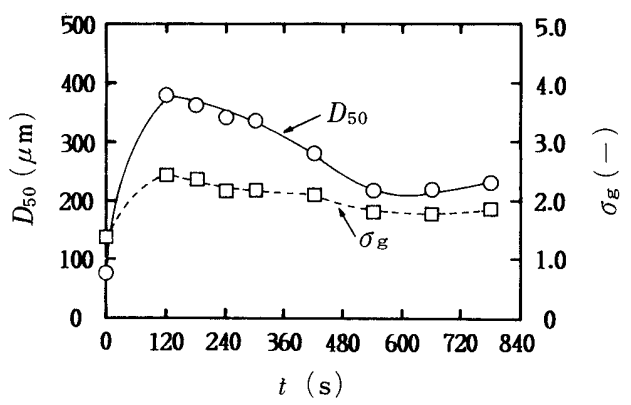

Fig. 4. Temporal Variation of Mean Particle Diameter and Geometric Standard Deviation

$\bigcirc$, mean particle diameter, $D_{50} ; \square$, geometric standard deviation, $\sigma_{\mathrm{g}}$.

Figure 4 indicates the plots of mean particle diameter and geometric standard deviation versus granulation time. From this figure, the granulation process was explained as follows. As soon as the binding solution was added to the powder materials, agglomerates were constructed. Although most of the powder materials were sparingly soluble in water, the binding solution added was distributed on the surface of the particles without permeating them, thus the coarse agglomerates were produced in the initial stages. Because of the shearing and tumbling effect of the blade, the agglomerates were gradually cut down to pieces, and the particle size distribution was also made to be narrow. At $t=540 \mathrm{~s}$, the $D_{50}$ reached to the minimum value with sharp particle size distribution. Although a slight granule growth was found, it appeared almost constant.

From these findings, it was concluded that the C.V. value and the intensity of the spectrum showed a large value in which the granule growth was remarkable and the geometric standard deviation was large. In this case, due to the wide particle size distribution and the unevenness of the particle shape, the impacted force to the blade was distributed, and resulted in the large value of fluctuation of power consumption. In the phase where the granule growth was gentle with sharp particle size distributions, due to the evenness of the impacting force, fluctuation of power consumption was small. Therefore, the granulation end point which showed the steady state of granule growth was determined where the C.V. value and the intensity of the spectrum showed the minimum value.

\section{Conclusion}

The frequency analysis and the computation of C.V. value 
based on the power consumption data measured during the agitation granulation process was performed using the sparingly soluble acetaminophen as a component in the powder materials, and we obtained the following, results. 1) A practical method for determination of granulation end point is applicable to the powder materials with sparingly soluble acetaminophen, thus the universality of this method is proved. 2) The granulation process of the wet ag- glomeration in the case of acetaminophen is discussed on the basis of the frequency analysis and the computation of the C.V. value of the power consumption.

\section{References}

1) K. Terashita, S. Watano and K. Miyanami, Chem. Pharm. Bull., 38, 3120 (1990).

2) K. Terashita, M. Katou, A. Ohike and K. Miyanami, Chem Pharm. Bull., 38, 1977 (1990). 